whatever reason, to disclose their management strategies to fellow professionals. Guidelines may be left unread, management protocols unheeded, and audit avoided. Patients and their relatives have no yardstick with which to measure the standard of care. Dreading a painful and undignified death, they often remain ignorant of available options.

Unnecessary suffering is likely as long as there is confusion in the lay mind between euthanasia and palliation and doctors fail to acknowledge that disease other than malignancy may require palliation. With a few exceptions, hospices currently cater for patients with a diagnosis of malignancy, ${ }^{1}$ but general practitioners can extend palliative care to wider groups. Ideally, clinicians whose task is to cure will have the wisdom to know when to change direction, and primary care teams will no longer regard palliative care as being "peripheral to their workload." ${ }^{2}$ Failure to treat the distress of terminal respiratory disease, for example, has its roots in deeply entrenched undergraduate training: opiates may "depress the respiratory centre"- but so much more is at stake than this naked physiological truth.

Identifying and correcting poor practice are no easier in palliative care than in any other branch of medicine. Exposing ignorance is never comfortable. Sadly, critical self awareness and the ability to live with uncertainty and imperfection are not yet on the curriculum of all our medical schools (although many would agree that this is what medical education should be about).

There may come a time when a diploma in palliative care is a necessary qualification for aspiring general practitioners and all patients will have access to comprehensive community based services. The availability of such services, however, depends on the decisions of commissioners who may lack appropriate experience. Academic departments of general practice have much to offer (not least in the development of evaluative tools) and are a valuable resource for both those who plan and those who deliver community care. The royal college's report is an ambitious, honest document full of enthusiasm for the monumental task of supporting the principles of good palliative care and effective teamwork in the community. It makes encouraging reading.

ZOE KENYON

Ebbor House

Hythe,

Kent, CT21 4BY

1 Royal College of General Practitioners. General practice palliative care facilitator project. Report of a evaluation. London: RCGP, 1995.

2 Saunders C, Walsh TD, Smith M. Hospice care in motor neurone disease. In: Saunders C, Summer DH, Teller N, eds. Hospice: the living idea. London: Edward Arnold, 1981:126-55.

\title{
Chemotherapy for non-small cell lung cancer
}

\section{A meta-analysis suggests that the benefits are small}

For some cancers, treatments are so effective that the question of whether to treat does not arise. For many others, however, while gratifying responses sometimes occur, there are also substantial toxicities related to treatment, and benefits of any kind may be small. The toxicities, inconvenience, and expense of chemotherapy are endured by both patients whose tumours do and do not respond. When faced with such imperfect treatments, clinical trialists must determine, within the limitations of biological variability, whether these treatments result in statistically significant benefits and at what cost. Doctors then have to decide whether these benefits are clinically important and whether they outweigh potential risks for a particular patient.

With the relatively small absolute survival benefits observed for chemotherapy of non-small cell lung cancer, large numbers of patients are required to draw conclusions with confidence. In this issue of the Journal Albertini et al report the results of a meta-analysis of updated data from 9387 patients participating in 52 randomised clinical trials comparing chemotherapy with no chemotherapy in non-small-cell lung cancer ( $p$ 899). ${ }^{1}$ They analysed trials in three clinically relevant disease settings: early, locally advanced, and advanced disease. In early disease treated with surgery and chemotherapy, mortality was $15 \%$ higher in patients treated with alkylating agents (treatments in vogue 10 years ago). In contrast, significant survival benefits were found among patients in several of the subgroups given more modern regimens containing cisplatin.

How large were the benefits? When combined with surgery or radical radiation in early or locally advanced disease, chemotherapy increased survival at two and five years (deaths fell by $13 \%$ in the radiotherapy and chemotherapy groups). In advanced disease chemotherapy produced $27 \%$ fewer deaths than in the group managed with best supportive care, equiva- lent to a $10 \%$ absolute improvement in survival at one year. The median survival, however, was prolonged by only 1.5 months.

While statistically significant, these benefits have only modest clinical impact. But given the number of people with lung cancer, these results indicate that, if chemotherapy were applied to appropriate patients, tens of thousands of people world wide would be alive at one year (or two and five years in patients eligible for radical radiotherapy) who would not be alive without chemotherapy. This extrapolation confers another level of importance to these findings. In fact, the size of the effect when compared with that of best supportive care is equivalent to that observed for adjuvant chemotherapy for breast cancer with spread to the nodes, which is considered to be the standard of care for that disease. ${ }^{2}$ Nevertheless the aggressive nature of lung cancer, with its propensity for early metastases, suggests that only a subset of patients with nonsmall cell lung cancer may benefit from chemotherapy.

Any mechanism allowing the identification of this chemotherapy responsive subset could dramatically improve the risk benefit ratio of chemotherapy for the entire population. Interestingly, for all the groups analysed in this paper, there was no evidence that any group specified by age, sex, histological findings, performance status, or stage benefited more or less from chemotherapy. It should be noted, however, that participants enrolled in randomised trials are usually fully ambulatory, and thus the data derived from this study, apply only to this subset. Molecular biological characteristics of the tumour (for example, p53 immunostaining, ras mutation, $b c l-2$, and Her $2 / n e u$ overexpression or as yet undiscovered markers) may provide part of the answer as well. ${ }^{34}$ We suggest that the question of testing for drug sensitivity in vitro should be reopened as resistance of many tumours in vitro seems to correlate with resistance in vivo. ${ }^{56}$ 
The findings of this meta-analysis (that benefit accrues from regimens containing cisplatin, while alkylating agents produce adverse effects) suggest that further benefits may emerge from continued studies of newer, even more active agents but that care must be used in selecting these drugs. Hopefully, this publication will stimulate further randomised controlled trials of promising new drugs. These include taxanes (paclitaxel and docetaxel), camptothecins (topotecan and CPT-I 1), new antimetabolites (such as gemcitabine and difluorodeoxycytidine), and antitubulin agents (vinorelbine). We also need studies to determine whether particular molecular characteristics of tumours identify subsets of patients likely to benefit from chemotherapy.

The applicability of these findings to current clinical practice outside the context of clinical protocols is less clear. In certain categories of patients, off protocol treatment can clearly be supported by the data presented, particularly when chemotherapy is used in combination with radical radiotherapy for locally advanced disease-this study shows highly significant differences in overall survival at both two and five years. The larger reduction in risk and the high level of significance in the patients with advanced disease is provocative, but the relatively small absolute additional survival provided by chemotherapy tempers our enthusiasm for treating these patients with chemotherapy. Such treatment should be limited to motivated patients with good performance status, who not only understand the risks and benefits associated with treatment but also have disease that can be readily evaluated. Treatment can therefore be stopped quickly if it proves ineffective. For patients with early disease treated surgically the evidence does not suggest that postoperative chemotherapy alone can be recommended. Exciting new data on preoperative ("neoadjuvant") chemotherapy suggest a possible way forward..$^{78}$

DAVID P CARBONE Associate professor JOHN D MINNA Professor

Simmons Cancer Center,

University of Texas Southwestern Medical Center,

Dallas,

Texas,

USA

1 Non-small Cell Lung Cancer Collaborative Group. Chemotherapy in non-small cell lung cancer: a meta-analysis using updated data on individual patients from 52 randomised trials. $B M F$ 1995;311:899-909.

2 Bonadonna G, Valagussa P, Moliterni A, Zambetti M, Brambilla C. Adjuvant cyclophosphamide, methotrexate, and fluorouracil in node-positive breast cancer: the results of 20 years of follow-up. NEngI F Med 1995;332:901-6.

3 Tsai CM, Chang KT, Perng RP, Mitsudomi T, Chen MH, Kadoyama C, et al. Correlation of intrinsic chemoresistance of non-small cell lung cancer cell lines with HER-2/neu gene expression but not with ras gene mutations. I Natl Cancer Inst 1993;85:897-901.

4 Muss HB, Thor AD, Berry DA, Kute T, Liu ET, Koerner F, et al. c-erbB2 expression and response to adjuvant therapy in women with node-positive early breast cancer. $N$ Engl $\mathcal{F} \mathrm{Med}$ 1994;330:1260-6.

5 Gazdar AF, Kadoyama C, Venzon D, Park JG, Tsai CM, Linnoila RI, et al. Association between histological type and neuroendocrine differentiation on drug sensitivity of lung cancer cell lines. Monogr Natl Cancer Inst 1992;13:191-6.

6 Tsai CM, Ihde DC, Kadoyama C, Venzon D, Gazdar AF. Correlation of in vitro drug sensitivity testing of long-term small cell lung cancer cell lines with response and survival. Eur $f$ Cancer 1990;26:1148-52.

7 Roth JA, Fossella F, Komaki R, Ryan MB, Putnam J Jr, Lee JS, et al. A randomised trial comparing perioperative chemotherapy and surgery with surgery alone in resectable stage III A non-small cell lung cancer [see comments]. $\mathcal{F}$ Natl Cancer Inst 1994;86:673-80.

8 Rosell R, Gomez-Codina J, Camps C, Maestre J, Padille J, Canto A, et al. A randomised trial comparing preoperative chemotherapy plus surgery with surgery alone in patients with non-small cell lung cancer. $N$ Engl f Med 1994;330:153-8.

\section{Defining ischaemia}

\section{When cells start screaming for help}

Ischaemia is a Greek-Latin word that means a stopping or restraint of the blood. ${ }^{1}$ Last December's issue of Cardiovascular Research published 33 responses by "eminent cardiologists" to the question "Myocardial ischaemia: can we agree on a definition for the 21 st century?" The short definitions included: "supply-demand imbalance," "blood supply inadequate to meet the energy needs of the heart," "coronary flow is insufficient to cope with the oxygen demand," and "coronary flow is incapable of delivering enough oxygen to support mitochondrial oxidation."

Concluding the section, the then editor of Cardiovascular Research, David Hearse, proposed that "biochemical ischaemia" exists when coronary blood flow is inadequate to permit the maintenance of a steady state metabolism, while "physiological ischaemia" exists when coronary flow is inadequate to permit the organ to perform at a level sufficient to support the body over its full physiological range of activity. While not wanting to add to the traditional definitions given by these experts, I want to present a functional view of ischaemia and specifically what happens to the ion channels of myocardial cells.

In my contribution I jokingly defined ischaemia as when the myocardial cells in a region of the heart start screaming for help. In other words, ischaemia is when affected myocardial cells invoke various mechanisms to protect themselves from calcium overload and necrosis. ${ }^{1}$ These protective mechanisms mainly involve two types of ion channels in the cell membrane: the slow calcium channel and the potassium channel which is regulated by ATP.
Myocardial cells' most important protective response to ischaemia (for example, during a transient vasospasm) revolves around the special properties of the slow calcium channels. ${ }^{23}$ It is calcium influx into the myocardial cells during excitation, occurring primarily through the slow calcium channels, which are activated by voltage, that controls the force of contraction. This calcium influx releases more calcium from stores in the sarcoplasmic reticulum. The resultant rise in intracellular free calcium concentration activates the contractile proteins. This process is the essential part of excitation-contraction coupling, with calcium ions as the link between excitation and contraction.

Myocardial cells must closely regulate the concentration of intracellular calcium. Several mechanisms achieve this, including regulation of calcium influx by the activity of the slow calcium channels, regulation of the activity of at least one type of potassium channel, the sodium-calcium exchange reaction, and the calcium ATPase/pump.

Slow calcium channels have several special properties. These include rapid and reversible inhibition by local acidosis, ${ }^{45}$ dependence on ATP for activity, ${ }^{6-8}$ and relatively selective inhibition by calcium antagonist drugs (such as verapamil, nifedipine, and diltiazem). Half inhibition of the slow calcium channels occurs at a $\mathrm{pH}$ of about $6 \cdot 6$, with complete inhibition at about $\mathrm{pH} 6 \cdot 1 .{ }^{4}$ As an ischaemic region of the heart becomes rapidly acidotic the calcium channels are relatively selectively inhibited. Calcium influx and the force of contraction of the afflicted cells therefore fall (acidosis also renders contraction proteins less sensitive to calcium, further 